\title{
A new formulation for the ionospheric cross polar cap potential including saturation effects
}

\author{
A. J. Ridley \\ University of Michigan, Ann Arbor, Michigan, USA
}

Received: 28 February 2005 - Revised: 18 October 2005 - Accepted: 20 October 2005 - Published: 21 December 2005

\begin{abstract}
It is known that the ionospheric cross polar cap potential (CPCP) saturates when the interplanetary magnetic field (IMF) $B_{z}$ becomes very large. Few studies have offered physical explanations as to why the polar cap potential saturates. We present 13 events in which the reconnection electric field (REF) goes above $12 \mathrm{mV} / \mathrm{m}$ at some time. When these events are examined as typically done in previous studies, all of them show some signs of saturation (i.e., over-prediction of the CPCP based on a linear relationship between the IMF and the CPCP). We show that by taking into account the size of the magnetosphere and the fact that the post-shock magnetic field strength is strongly dependent upon the solar wind Mach number, we can better specify the ionospheric CPCP. The CPCP $(\Phi)$ can be expressed as $\Phi=\left(10^{-4} v^{2}+11.7 B\left(1-e^{-M_{a} / 3}\right) \sin ^{3}(\theta / 2)\right) r_{m s} / 9$ (where $v$ is the solar wind velocity, $B$ is the combined $Y$ and $Z$ components of the interplanetary magnetic field, $M_{a}$ is the solar wind Mach number, $\theta=a \cos \left(B_{z} / B\right)$, and $r_{m s}$ is the standoff distance to the magnetopause, assuming pressure-balance between the solar wind and the magnetosphere). This is a simple modification of the original Boyle et al. (1997) formulation.
\end{abstract}

Keywords. Ionosphere (Electric fields and currents; Polar ionosphere) - Magnetospheric physics (Solar windmagnetosphere interactions)

\section{Introduction}

It has been shown in a number of studies that many ionospheric electrodynamic properties can be described as being linearly related to the interplanetary magnetic field (IMF) and solar wind velocity. For example, Papitashvili et al. (1994) and Friis-Christensen et al. (1985) show that ground magnetic perturbations can be linearly related to the IMF $B_{z}$ and $B_{y}$ components. These relationships can be com-

Correspondence to: A. J. Ridley

(ridley@umich.edu) bined with an ionospheric conductance pattern to determine a linear relationship between the IMF and the ionospheric potential pattern. Ridley et al. (2000) use the assimilative mapping of ionospheric electrodynamics (AMIE) technique (Richmond and Kamide, 1988) to calculate a large number of ionospheric electric potential maps from ground-based magnetometers and show that the potentials are linearly related to the IMF $B_{z}$ and $B_{y}$. Papitashvili and Rich (2002) show that electric potentials derived from in-situ measurements of the ionospheric plasma flow also shows a linear relationship to the IMF. Most of the above analysis was completed for small magnitude IMF time periods.

Only a few studies have attempted to examine the saturation that may occur under strong driving of the solar wind and IMF. Reiff et al. (1981) compare in-situ measurements of ionospheric plasma flow (or electric fields and the resulting potential) to different magnetospheric coupling functions (such as the Kan and Lee, 1979, function). They find that using an amplified magnetic field (due to the bow shock compression) works best, but that the amplified field has to be limited to get the best fits. The best amplification factor is $7-8$, with a limiting value of $\sim 60$ (corresponding to a maximum IMF of $\sim 8 \mathrm{nT}$ ).

Weimer et al. (1990) investigate the saturation of the auroral electrojet (AE) index to both the IMF $B_{z}$ and the solar wind velocity $(V)$ multiplied by $B_{z}$. They show that the maximum $\mathrm{AE}$ reaches a saturation value at $B_{z}=-15 \mathrm{nT}$, or $V B_{z}=-8 \mathrm{mV} / \mathrm{m}$. They further point out that the AE index has been related to the cross polar cap potential (CPCP) (Ahn et al., 1984), so this indicates that the CPCP most likely saturates at similar values.

Russell et al. (2000) examine the high-latitude ionospheric electric potential and Joule heating saturation during the 24 25 September 2000 storm. They attempt to show that the high-latitude features saturate while the ring current injection rate does not. They further argue that the saturation takes place when the solar wind velocity times $B_{z}$ (i.e., the $Y$ component of the interplanetary electric field, or IEF) reaches a level of $3 \mathrm{mV} / \mathrm{m}$. This is an equivalent magnetic field $B_{z}$ of 
$7.5 \mathrm{nT}$ with a solar wind speed of $400 \mathrm{~km} / \mathrm{s}$ (similar to the Reiff et al., 1981, value). Russell et al. (2001) show five time periods which arguably show signs of saturation in the potential and Joule heating. They continue to state that the saturation occurs near an IEF of $3 \mathrm{mV} / \mathrm{m}$. Liemohn and Ridley (2002) take issue with the claims of saturation stated by Russell et al. (2001). They argue that the presented events can be fit with a linear function with similar error, and that the saturation occurs closer to $10 \mathrm{mV} / \mathrm{m}$.

Nagatsuma (2002) shows that, on a statistical basis, the saturation occurs around $5 \mathrm{mV} / \mathrm{m}$ (or a $B_{z}$ of $12.5 \mathrm{nT}$ ). He includes all available data from 1995-1999, which was during solar minimum and the rise to solar maximum. From Fig. 3 in their study, there is an indication that the relationship may be more complicated than simple saturation - there is a huge scatter in the points above $5 \mathrm{mV} / \mathrm{m}$. The Nagatsuma (2002) study uses the polar cap index (PCI) as a proxy for the CPCP. Troshichev et al. (1996) show that the PCI can be related to the ionospheric potential by $\Phi=19.35 P C I+8.78$, where $\Phi$ is the ionospheric CPCP in $\mathrm{kV}$. Ridley and Kihn (2004) also show that the PCI is linearly related to the CPCP.

In Shepherd et al. (2002), the SuperDARN radar data indicate that the ionospheric potential saturates at an IEF of approximately $15-20 \mathrm{mV} / \mathrm{m}$. This study uses $163810-\mathrm{min}$ time periods in which there was very steady IMF and solar wind to show that the potential rarely reaches above $100 \mathrm{kV}$, which is much lower than other techniques indicate.

Siscoe et al. (2002) is one of the only studies that attempts to explain why the potential saturates. They argue that the saturation of the cross polar cap potential is an internal process - the region 1 currents flowing into the ionosphere tend to reduce the magnetic field near the subsolar magnetosphere, inhibiting reconnection. Equation (13) of the Siscoe et al. (2002) study relates the ionospheric cross polar cap potential $\Phi_{p c}$ (i.e. $\Phi$ above) to the electric field $\left(E_{S W}\right)$ and pressure $\left(p_{S W}\right)$ in the solar wind, the IMF clock angle $(F(\theta))$, the dipole strength $(D)$, a geometrical factor $(\xi)$, and the ionospheric conductance $\left(\Sigma_{0}\right)$ :

$\Phi_{p c}=\frac{57.6 E_{r} p_{s w}^{1 / 3} D^{4 / 3} F(\theta)}{p_{s w}^{1 / 2} D+0.0125 \xi \Sigma_{0} E_{r} F(\theta)}$

where $\Phi_{p c}$ is in $k V$. This formulation takes into account the compression of the magnetosphere due to the solar wind dynamic pressure, the reconnection efficiency, and those terms included directly above. Shepherd et al. (2003) show that in order to get the SuperDARN measurements of the saturation to match the Siscoe et al. (2002) formulation, the ionospheric conductance has to be 23 mhos, which is rather high. This could also indicate that the SuperDARN radar computed CPCP during saturated events may be too low.

The present study suggests that the saturation of the ionospheric potential may actually be an external process. We present ionospheric cross polar cap potentials derived from the assimilative mapping of ionospheric electrodynamics (AMIE) technique (Richmond and Kamide, 1988), using the output presented in Ridley and Kihn (2004). These AMIE runs were made with well over 100 magnetometers, and have been shown to reproduce ion flow measurements made by low altitude satellites, even during disturbed time periods (Bekerat et al. 2005; Kihn et al., 2005). There are inherent problems with computing the ionospheric cross polar cap potential with any technique: (1) With AMIE, using only magnetometers, one can argue that the patterns are strongly dependent upon the conductance. Indeed, this was pointed out by Ridley and Kihn (2004), who showed that there is a seasonal difference between the PCI estimated CPCP and the AMIE derived potential. It is unknown whether this is a problem with AMIE or the polar cap index. (2) Low-altitude satellites only measure a single slice of the potential pattern, so, it is thought that the measured CPCP is only a lower estimate, since the satellite may not pass through the maximum or minimum potential (Ober et al., 2003). In addition, since the satellites take $20 \mathrm{~min}$ to cross the pattern, the inferred potential may be a combination of different patterns. (3) Radars can not measure the entire pattern at one time, and the maximum and minimum in potential can go to lower latitudes than the radars, causing an underestimate of the potential (Shepherd et al., 2003).

Many of the events discussed here have been shown to be saturated in other studies before this one (e.g. Nagatsuma, 2002; Shepherd et al., 2002; Hairston et al., 2003; Siscoe et al., 2004). Russell et al. (2000, 2001), and Liemohn and Ridley (2002) also present AMIE results discussing the idea of the saturation of the cross polar cap potential, showing that AMIE can be used to describe the potential during strong driving.

One of the problems with examining how the saturation of the cross polar cap potential occurs is that there are only a few time periods in which the IMF is extremely large compared to the number of time periods in which the IMF is small. In order to overcome this difficulty, this study only examines time periods surrounding and including events in which the reconnection electric field (REF) exceeds $12 \mathrm{mV} / \mathrm{m}$. The REF is defined as by Sonnerup (1974) and Kan and Lee (1979):

$E_{r}=V B_{y z} \sin ^{2}(\theta / 2)$,

where $B_{y z}=\sqrt{B_{z}^{2}+B_{y}^{2}}$, and $\theta=\cos ^{-1}\left(B_{z} / B_{y z}\right)$, and $V$ is the solar wind speed.

The $12 \mathrm{mV} / \mathrm{m}$ limit allows an examination of the individual events and may allow a general cause for the saturation of the potential to be illuminated. In addition, $12 \mathrm{mV} / \mathrm{m}$ is four times the value suggested by Russell et al. (2001) and Reiff et al. (1981), less than twice the value suggested by Weimer et al. (1990), and just over the value suggested by Liemohn and Ridley (2002). This value is a good compromise between using more events because of a value too low and using few events because of a very high value; both of which would tend to skew the results.

There is also a question of what time-scales to use. The AMIE runs were conducted using a $1 \mathrm{~min}$ time-step. It is known that there are large errors propagating solar wind data 
Table 1. A summary of the values associated with the 13 events. The columns are the event starting date, the minimum IMF $B_{z}$ during the period, the minimum Alfvén Mach number, the RMS difference between the AMIE CPCP and the Boyle et al. (1997) formulation for the 48 hour period, the RMS error using Eq. (4), the decrease in the error using this formulation over the Boyle et al. (1997) formulation, the RMS error using Eq. (9), the decrease in the error over the Boyle et al. (1997) formulation, the RMS error using Eq. (9), but leaving out the $R_{m s} / 9$ term, and the decrease in the error using this formulation over the Boyle et al. (1997) formulation.

\begin{tabular}{lrrr|rrrrrrr}
\hline Date & $\begin{array}{r}\text { Min } B_{z} \\
\mathrm{nT}\end{array}$ & Min $M_{A}$ & $\begin{array}{r}\text { Boyle } \\
\mathrm{kV}\end{array}$ & \multicolumn{2}{c}{ Eq. (4) } & \multicolumn{2}{|c|}{ Eq. (9) } & \multicolumn{2}{|c|}{ Eq. (9) $-R_{m s} / 9$} \\
$\mathrm{kV}$ & $\mathrm{kV}$ & $\%$ & $\%$ \\
\hline 22 Sep. 1999 & -25.62 & 2.46 & 36.36 & 31.52 & 13.31 & 30.32 & 16.61 & 31.90 & 12.27 \\
21 Oct. 1999 & -30.98 & 1.80 & 71.58 & 63.98 & 10.61 & 34.81 & 51.36 & 37.11 & 48.16 \\
06 Apr. 2000 & -31.06 & 2.53 & 56.18 & 40.74 & 27.49 & 31.28 & 44.33 & 39.18 & 30.25 \\
23 May. 2000 & -36.31 & 1.66 & 44.85 & 36.46 & 18.70 & 31.73 & 29.24 & 37.05 & 17.40 \\
15 Jul. 2000 & -59.64 & 0.69 & 99.10 & 82.50 & 16.75 & 36.40 & 63.26 & 41.40 & 58.22 \\
11 Aug. 2000 & -29.60 & 1.53 & 60.75 & 59.49 & 2.08 & 36.80 & 39.43 & 36.12 & 40.54 \\
17 Sep. 2000 & -38.04 & 2.08 & 33.69 & 30.99 & 8.03 & 29.99 & 10.99 & 29.69 & 11.87 \\
04 Oct. 2000 & -27.30 & 2.58 & 44.50 & 39.24 & 11.83 & 28.21 & 36.61 & 29.58 & 33.54 \\
06 Nov. 2000 & -15.49 & 1.24 & 35.39 & 42.70 & -20.66 & 33.43 & 5.52 & 31.62 & 10.63 \\
30 Mar. 2001 & -48.82 & 1.03 & 119.64 & 88.95 & 25.65 & 43.95 & 63.26 & 63.21 & 47.16 \\
11 Apr. 2001 & -33.87 & 0.85 & 51.49 & 50.34 & 2.23 & 54.92 & -6.67 & 53.25 & -3.42 \\
02 Oct. 2001 & -23.41 & 1.26 & 46.27 & 49.13 & -6.19 & 25.58 & 44.72 & 27.01 & 41.62 \\
21 Oct. 2001 & -26.59 & 2.02 & 44.39 & 33.91 & 23.60 & 28.44 & 35.92 & 36.30 & 18.22 \\
\hline Total & & & & & 10.03 & & 33.42 & & 28.19 \\
\hline
\end{tabular}

from satellites to the magnetopause that can be on the order of $10 \mathrm{~min}$ or more (Ridley, 2000). It is further known that the ionospheric convection can change on time-scales of $12 \mathrm{~min}$ (Ridley et al., 1998). To balance the error of the propagation with the time-scales of changes in the potential, we averaged all of the data to $15 \mathrm{~min}$. This is the same resolution of the Polar Cap index data used in other studies (Nagatsuma, 2002, 2004). While this will tend to wash out the extremes in the potential, the overall time evolution of the potential should be well maintained. During many of the events, the IMF and solar wind have large-scale features that last for hours, implying that the potential should vary slowly through out the time periods.

This paper first suggests that using the Boyle et al. (1997) formulation as the expected potential is not physically accurate, since the length of the reconnection line is not considered. To account for this, the definition of the relationship between the potential and the solar wind and IMF is altered. The derived potential still over-predicts the AMIE derived potential in a few events. This implies that these events are saturated. It is then suggested that the solar wind Alfvén Mach number may influence the potential, so the Boyle et al. (1997) formulation is further altered to include the solar wind Alfvén Mach number. When this is done, the modeled and measured potential agree much better than without the modifications. It is suggested that the mechanism for the saturation of the ionospheric potential may be external to the mag- netosphere, and not an internal mechanism, as suggested by the Siscoe et al. (2002) study.

\section{Results}

Figures 1-4 show 13 periods in which the reconnection electric field (REF) becomes larger than $12 \mathrm{mV} / \mathrm{m}$ for some interval of time. For each event, we show the $B_{z}$ and $B_{y}$ components of the IMF, the cross polar cap potential from AMIE and the CPCP estimated by an analytical function that assumes a linear relationship between the IMF and the ionospheric potential. This linear analytical function is by Boyle et al. (1997):

$\Phi=10^{-4} v^{2}+11.7 B \sin ^{3}(\theta / 2)$,

where we took $B=B_{y z}, \theta$ as defined above, and $v=v_{x}$. Each cluster of plots also includes two scatter plots that demonstrate various ways in which the ionospheric cross polar cap potential can be shown to have saturation. The upper scatter plot shows the CPCP estimated by Boyle et al. (1997) and the CPCP from AMIE, both as a function of the reconnection electric field, as defined by Eq. (2). The lower scatter plot shows the AMIE CPCP versus the Boyle et al. (1997) estimated CPCP, with a line showing where they would be equal. In addition, there is an indication of the root mean squared (RMS) difference between the two estimates. 


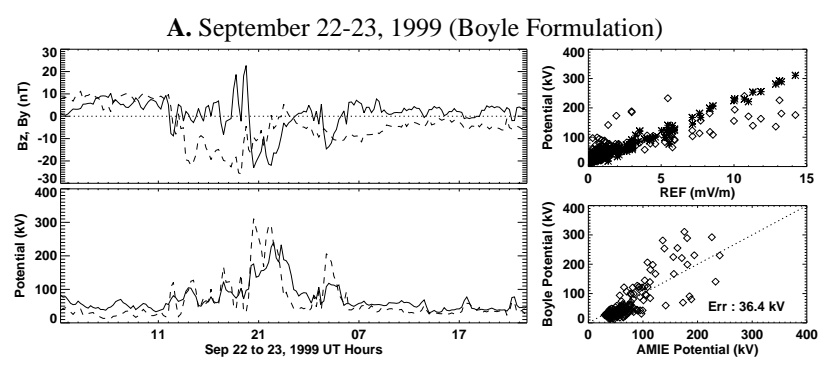

B. October 21-22, 1999 (Boyle Formulation)

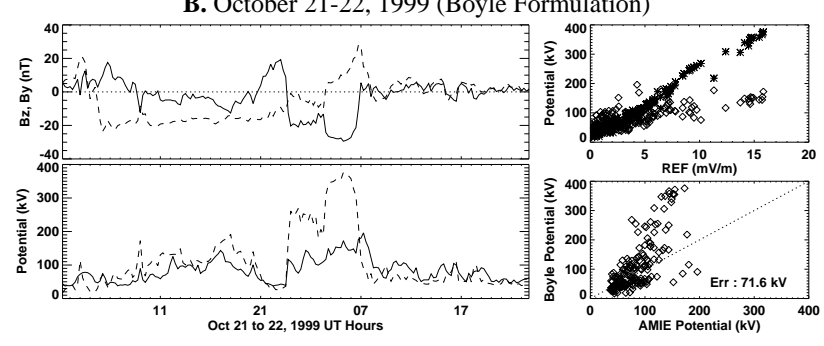

C. April 4-5, 2000 (Boyle Formulation)

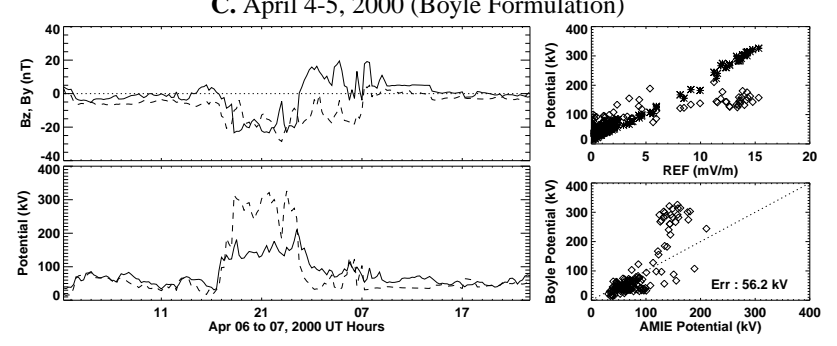

Fig. 1. The upper-left plot in each cluster shows the IMF $B_{z}$ (solid) and IMF $B_{y}$ (dashed). The lower-left plot shows the ionospheric cross polar cap potential (CPCP) as specified by AMIE (solid) and estimated from the Boyle et al. (1997) formulation (dashed). The upper-right plot shows the Boyle et al. (1997) (stars) and AMIE CPCP (diamonds) versus the reconnection electric field. The bottom-right plot show the AMIE CPCP versus the Boyle et al. (1997) formulation potential.

These clusters of plots show three different ways in which saturation of the ionospheric CPCP has typically been shown: (1) the time series plots show that the Boyle et al. (1997) estimation of the CPCP typically becomes larger than the AMIE CPCP when the IMF $B_{z}$ component becomes large and negative; (2) the upper scatter plot shows that the Boyle et al. (1997) estimated CPCP is linear for large REFs, while the AMIE CPCP is significantly lower than this linear estimate; and (3) when the Boyle et al. (1997) estimated and the AMIE CPCPs are plotted against each other, the Boyle et al. (1997) estimated potentials are much larger than the AMIE values. While the data are shown differently, each of these three plots show exactly the same thing: a linear relationship between the reconnection electric field and the ionospheric cross polar cap potential overestimates the potential when the REF becomes large. All of the events show this to be true. The exact value of the REF at which this overestimation starts to occur can be debated, but it is clear that it does occur in all of the events.
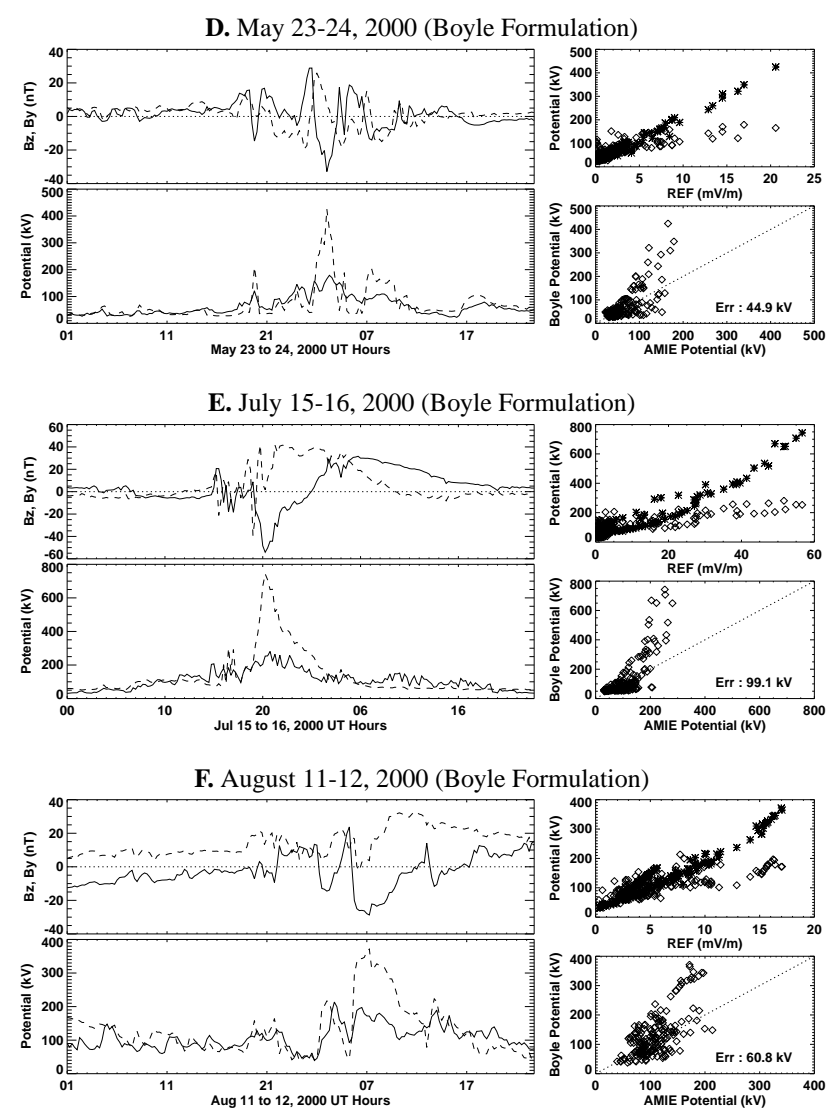

Fig. 2. Three more events in the same format as Fig. 1.

In most previous studies of the saturation of the cross polar cap potential (e.g. Russell et al., 2001; Merkine et al., 2003; Nagatsuma, 2002), they show plots such as Figs. 1-4, implying a relationship between an electric field and a potential. However there is something missing in this relationship - a length. An electric potential is the integral of an electric field along a path of some length. The above plots do not indicate any length scale at all.

This can be problematic, because when the reconnection electric field becomes large, often the solar wind density and velocity also become large. This compresses the magnetosphere, reducing the length-scale for the integration of the electric field. That implying that the cross magnetospheric potential could possibly decrease.

By modifying the Boyle et al. (1997) formulation to include a length scale, we can better relate the solar wind and IMF to the ionospheric CPCP. While the Boyle et al. (1997) formulation technically does not contain an electric field (since the first term only has a $v^{2}$, and the second term does not contain a velocity), it should still be dependent upon the size of the magnetosphere. Multiplying by a ratio of the size of the magnetosphere to a nominal size, this size dependence is achieved:

$\Phi=\left(10^{-4} v^{2}+11.7 B \sin ^{3}(\theta / 2)\right) \frac{r_{m s}}{9}$. 

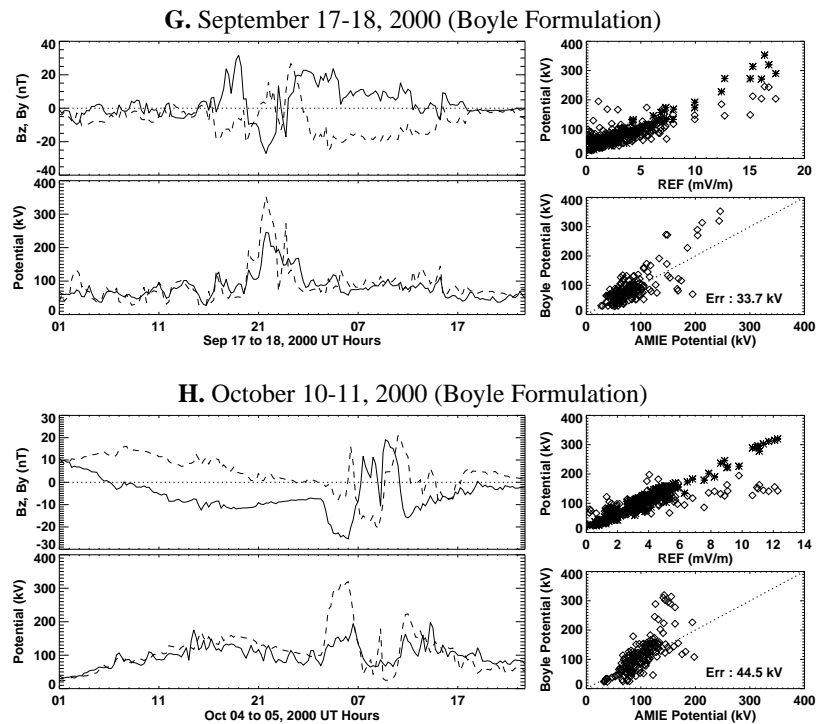

I. November 6-7, 2000 (Boyle Formulation)

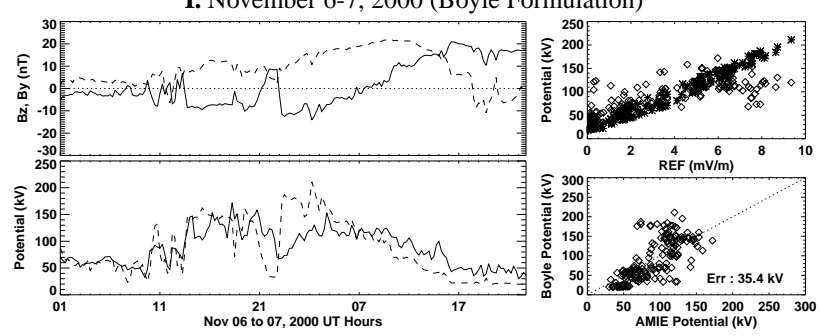

Fig. 3. Three more events in the same format as Fig. 1.

The radius of the magnetosphere $\left(r_{m s}\right)$ can be approximated through a pressure balance between the solar wind pressure and the magnetospheric magnetic field pressure (in $R_{e}$ ):

$r_{m s}=\left(\frac{\left(2 B_{s}\right)^{2}}{2 \mu_{0} P_{s w}}\right)^{1 / 6}$.

$B_{s}$ is the surface magnetic field, and $P_{s w}$ is the ram and magnetic pressure of the solar wind:

$P_{s w}=\frac{B^{2}}{2 \mu_{0}}+n M_{p} v^{2}$.

Where $N$ is the solar wind number density, $B$ is the magnitude of the IMF, and $M_{p}$ is the mass of a proton. Typically, the solar wind ram pressure $\left(n M_{p} v^{2}\right)$ is almost an order of magnitude larger than the magnetic pressure. In these extreme cases, though, the magnetic pressure can become comparable to the ram pressure, so it needs to be included. It should be noted that the radius of the magnetosphere along the Earth-Sun line has a seasonal dependence and a local time dependence because of the rotational axis tilt and the offset of Earth's dipole from the center of the planet. The effect on the radius of the magnetosphere should be less than about five percent, though. Furthermore, during periods in which the magnetic field in the solar wind becomes large, the shape of the magnetopause can be distorted (e.g. Raeder
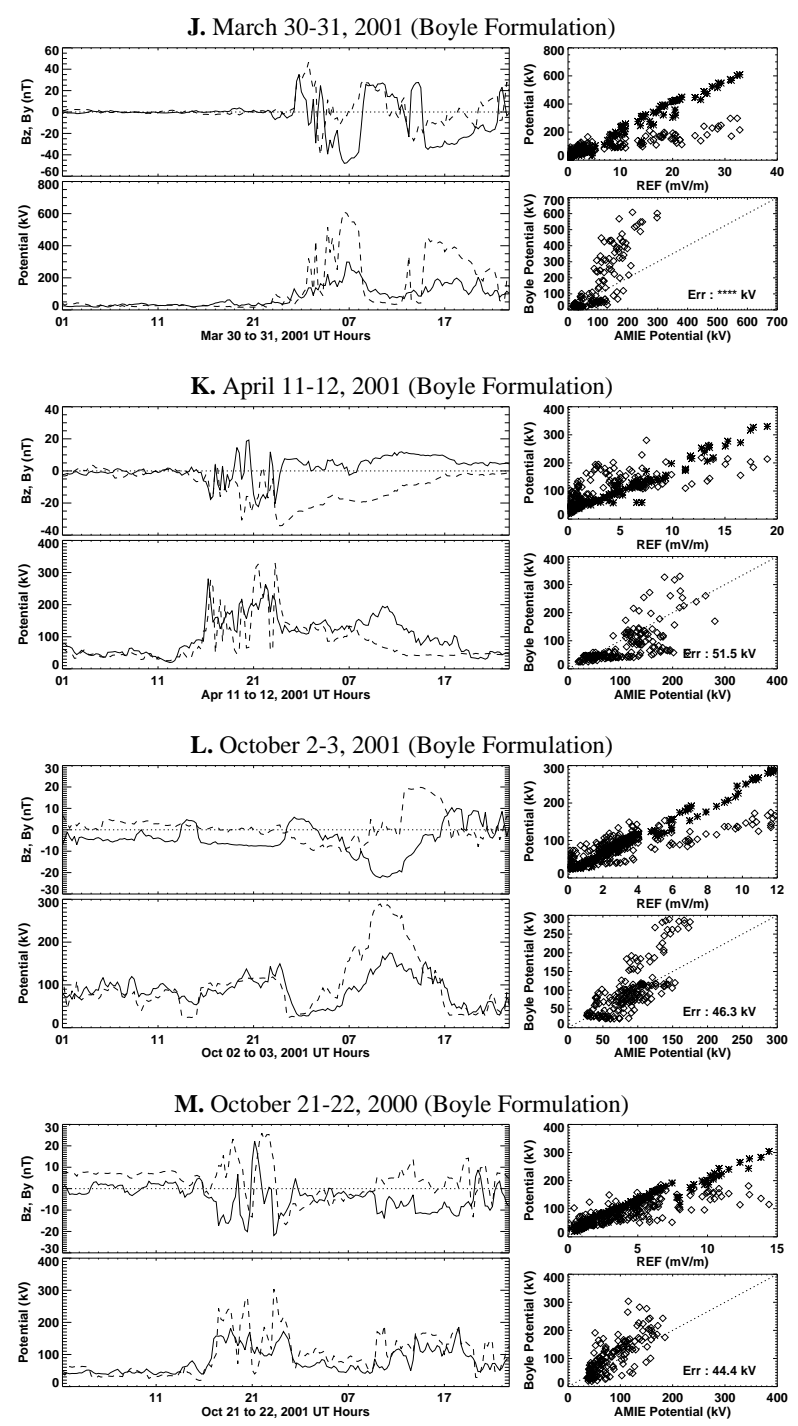

Fig. 4. Four more events in the same format as Fig.1.

et al., 2001; Siscoe et al., 2002, 2004). Although this distortion should be taken into account, it is most likely highly dependent on the direction of the IMF (i.e., parallel versus perpendicular shocks), and therefore one would need a global magnetospheric model to do this. Because we are not using a large-scale model of the magnetosphere in this research, it is beyond the scope of the current study.

In addition, Alpha particles are not used in the calculation of the solar wind pressure. At times, the Alpha particle population can exceed $10 \%$. Because they are four times heavier than the protons, they can actually account for $40 \%$ of the pressure. This $40 \%$ error would cause an error in the magnetopause stand-off distance of approximately $5.5 \%$.

Figures 5-8 show the pressure balanced radius of the magnetosphere, the AMIE CPCP and the estimated potential using Eq. (4). In addition, the RMS differences between the AMIE potentials and Eq. (4) estimated potentials are displayed on the plots. Table 1 summarizes all of the errors 
A. September 22-23, 1999 (Equation 4)
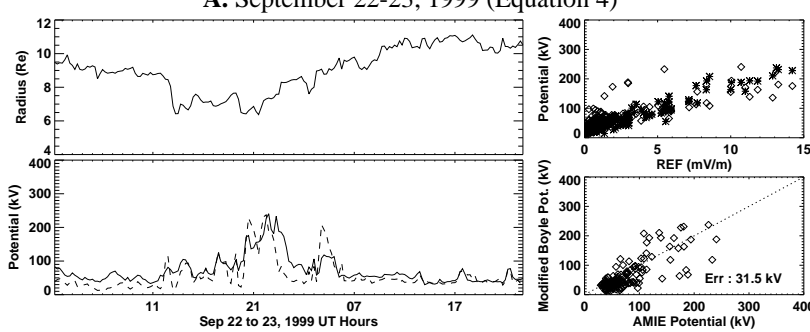

B. October 21-22, 1999 (Equation 4)

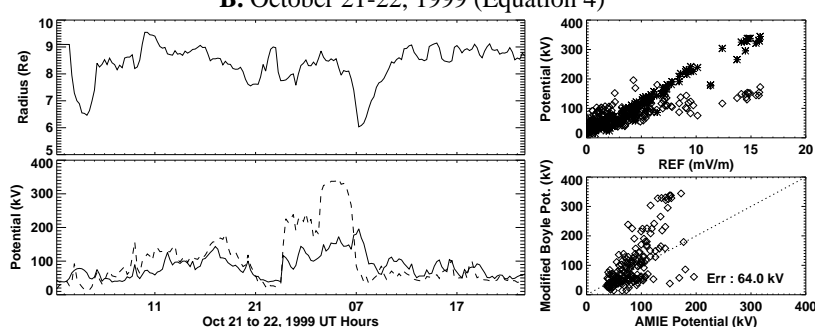

C. April 4-5, 2000 (Equation 4)

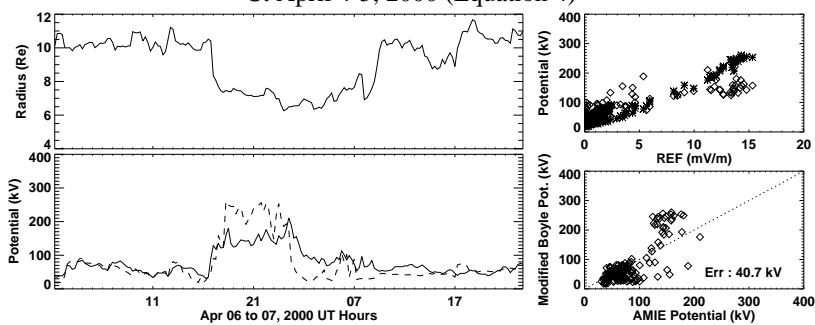

Fig. 5. The same three events in Fig. 1, plotted in the same way, except Eq. (4) was used rather than estimating the CPCP with Boyle et al. (1997). The top plot is the radius of the magnetosphere, as estimated by Eq. (5).

associated with all of the events. The percentage differences that are shown in the table are changes in the performance compared to the Boyle et al. (1997) formulation. The RMS difference between the potentials decreases by $10 \%$ over the Boyle et al. (1997) formulation when the magnetospheric size is considered.

Figures 5-8 compared to Figs.1-4 show a decrease in the amount of over-prediction of the ionospheric potential in most of the events. Some of these events show very little saturation at all (22 September 1999; 17 September 2000; 11 April 2001; 21 October 2001), implying that considering the radius of the magnetosphere may be important when examining large IMF and solar wind events. Some events (namely 21 October 1999; 15 July 2000; 30 March 2001; and 2 October 2001) still show significant over-predictions of the CPCP. It is evident that a modified formulation must be determined that further takes into account the saturation of the potential.

Let us consider a single event chosen at random, 30-31 March 2001. Figure 9 shows all of the relevant quantities for this time period, such as the reconnection electric field and the radius modification factor $\left(r_{m s} / 9\right.$, as discussed above). The third plot shows the solar wind Alfvén Mach number,

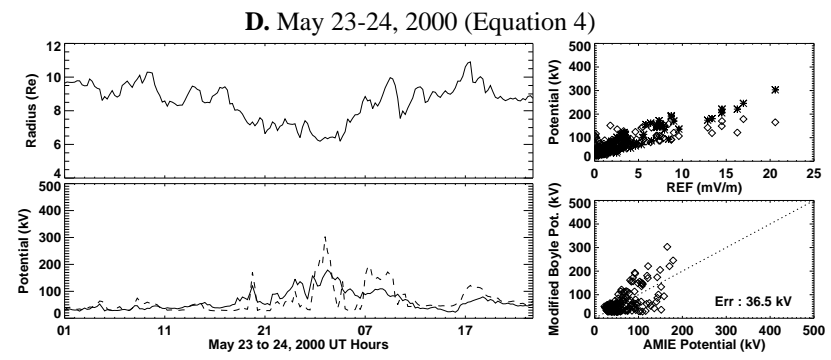

E. July 15-16, 2000 (Equation 4)

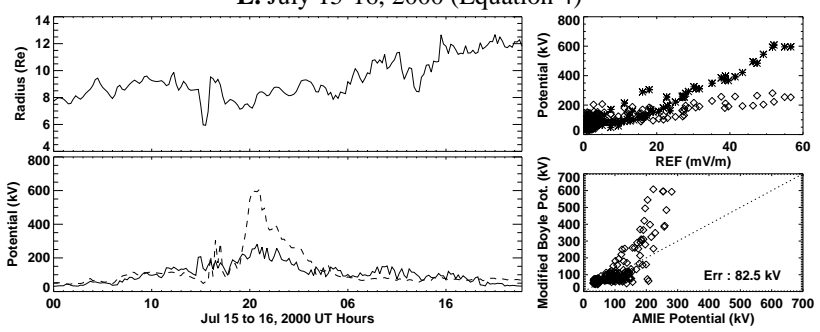

F. August 11-12, 2000 (Equation 4)

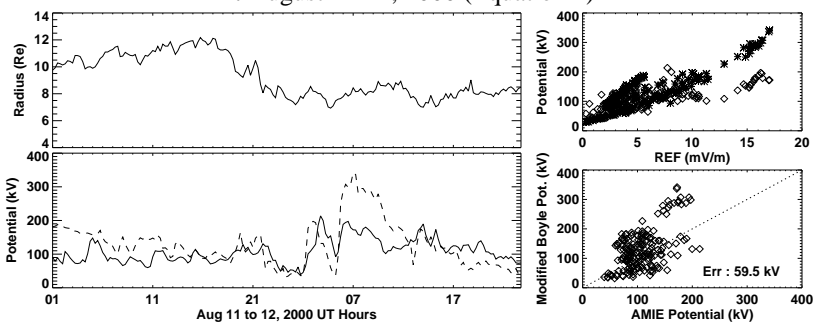

Fig. 6. The same three events in Fig. 2, plotted in the same way as Fig. 5.

which is defined as:

$M_{a}=\frac{V_{s w}}{C_{a}}$,

where

$C_{a}=\frac{B}{\sqrt{\mu_{0} n M_{p}}}$

The fourth plot shows the AMIE CPCP as well as the CPCP estimated from Boyle et al. (1997), while the fifth plot shows the AMIE and Eq. (4) estimated CPCPs. It is interesting to note that when the potential is saturated (i.e., the predicted potential is significantly larger than the AMIE value), the Alfvén Mach number is less than four. Indeed, this is true of all other events, and could in fact be the cause of the saturation.

Taking the Alfvén Mach number into consideration, we can express the ionospheric cross polar cap potential as:

$\Phi=\left(10^{-4} v^{2}+11.7 B\left(1-e^{-M_{a} / 3}\right) \sin ^{3}(\theta / 2)\right) \frac{r_{m s}}{9}$.

The term $\left(1-e^{-M_{a} / 3}\right)$, which multiplies the magnetic field, will be justified below, when the physics of the bow shock is discussed. When the Alfvén Mach number is in its typical range (approximately eight), the last term is 0.93 , 


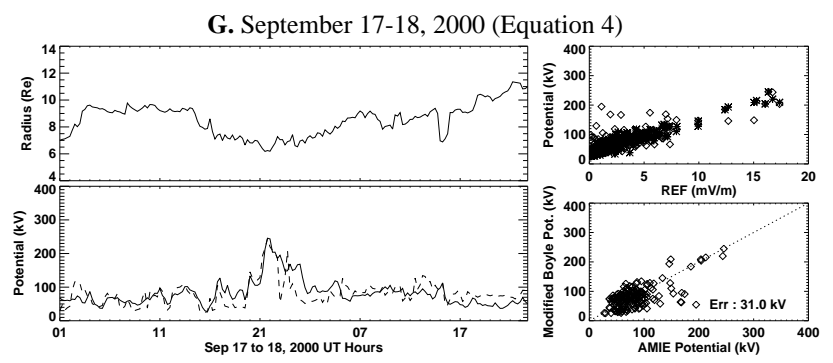

H. October 10-11, 2000 (Equation 4)

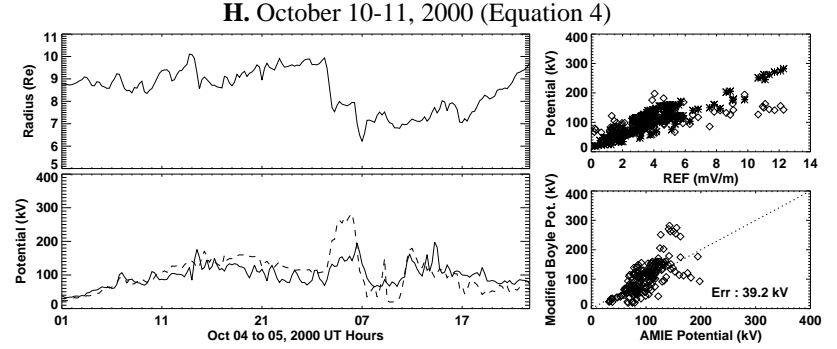

I. November 6-7, 2000 (Equation 4)

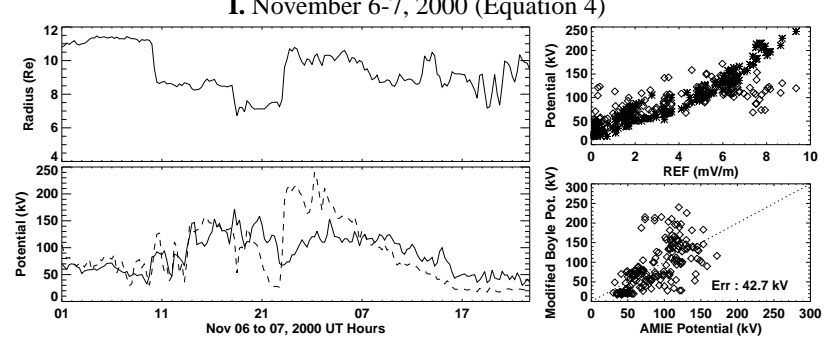

Fig. 7. The same three events in Fig. 3, plotted in the same way as Figure 5.

so it does not modify the potential very much at all. As the Mach number decreases significantly (i.e. the magnetic field becomes very large, or the solar wind density decreases dramatically - both of which occur in magnetic clouds) the last term decreases. At a Mach number of three, the term is 0.63 , and at a Mach number of one, it is 0.28 . The effect of this term on the 30-31 March 2001 event is shown in the sixth plot in Fig. 9. For comparison, the bottom plot in Fig. 9, shows the event using Eq. (9), but without the magnetospheric size correction factor $\left(r_{m s} / 9\right)$. The smallest error between the AMIE CPCP and the other formulations occurs when Eq. (9) is used.

Figure 10 shows examples of how this term modifies the ionospheric potential as a function of the reconnection electric field, solar wind velocity and solar wind density. The plots on the left have an input solar wind velocity of $400 \mathrm{~km} / \mathrm{s}$, while those on the right have an input velocity of $800 \mathrm{~km} / \mathrm{s}$. From top to bottom, the input solar wind densities are 1, 5, and $20 \mathrm{~cm}^{-3}$. These plots show that as the solar wind number density and velocity increase, the saturation of the potential occurs at a much higher REF.

When Eq. (9) is applied to all of the events, Figs. 11-14 result. These plots show the Alfvén Mach number and the estimated potential using Eq. (9). The saturation is reproduced in almost all cases, and strongly suggests that the saturation

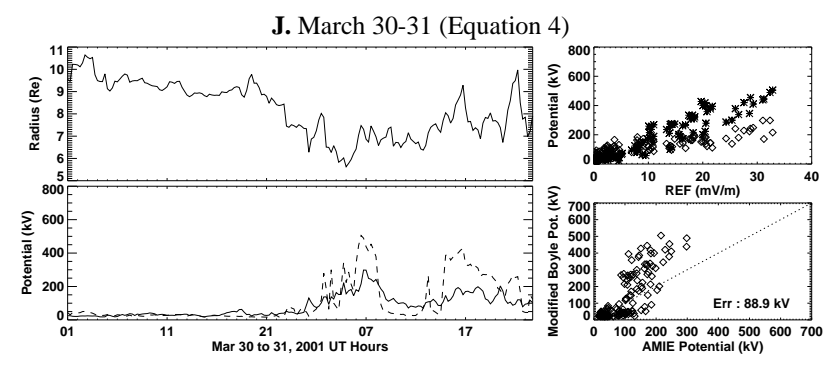

K. April 11-12, 2001 (Equation 4)
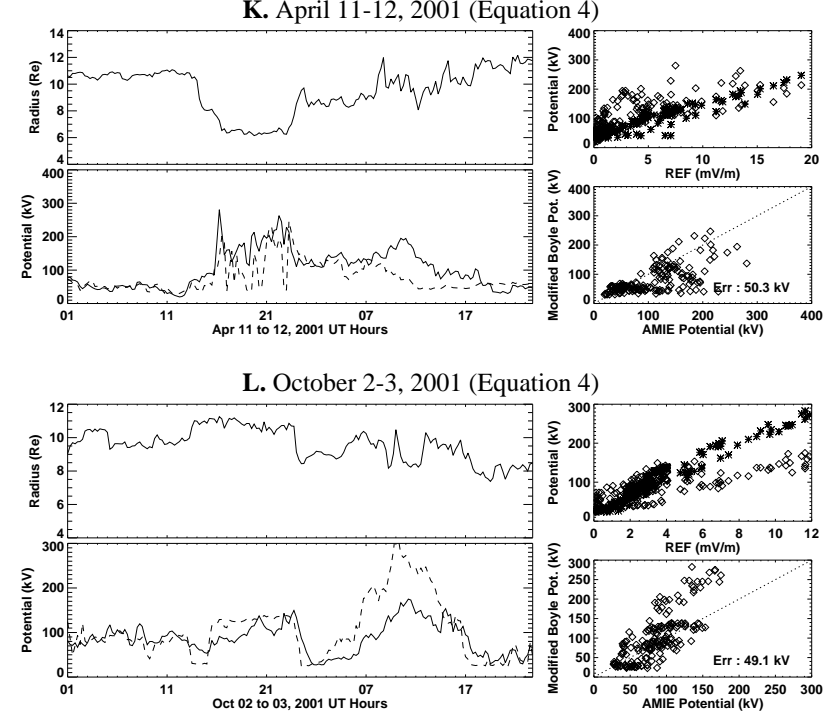

M. October 21-22, 2001 (Equation 4)

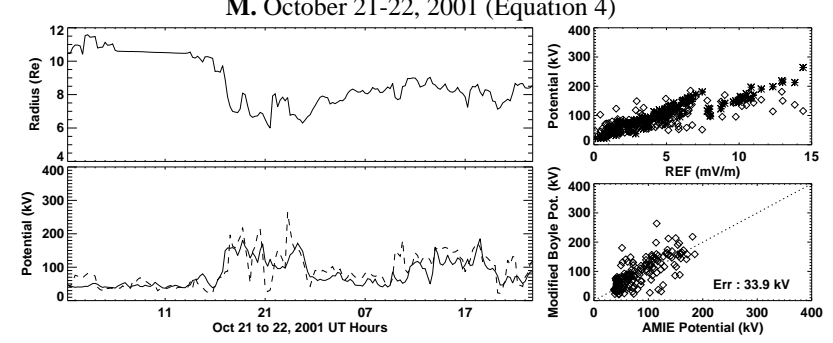

Fig. 8. The same four events in Fig. 4, plotted in the same way as Fig. 5.

may be tied to the Alfvén Mach number. The RMS difference is decreased by $33.4 \%$ over using simply the Boyle et al. (1997) formulation (see Table 1). Eq. (9) is a much better estimation of the cross polar cap potential than the Boyle et al. (1997) formulation during strong driving conditions.

\section{Discussion}

The study by Reiff et al. (1981) discusses the fact that when the IMF is advected through the bow shock, it increases in strength. For a typical solar wind density and flow speed, the IMF can increase by a factor of four from the solar wind to the magnetosheath. In addition, as the magnetic field in the solar wind becomes larger, the ratio between the shocked magnetic field and upstream field decreases. This is because the Alfvén Mach number decreases with increasing magnetic 


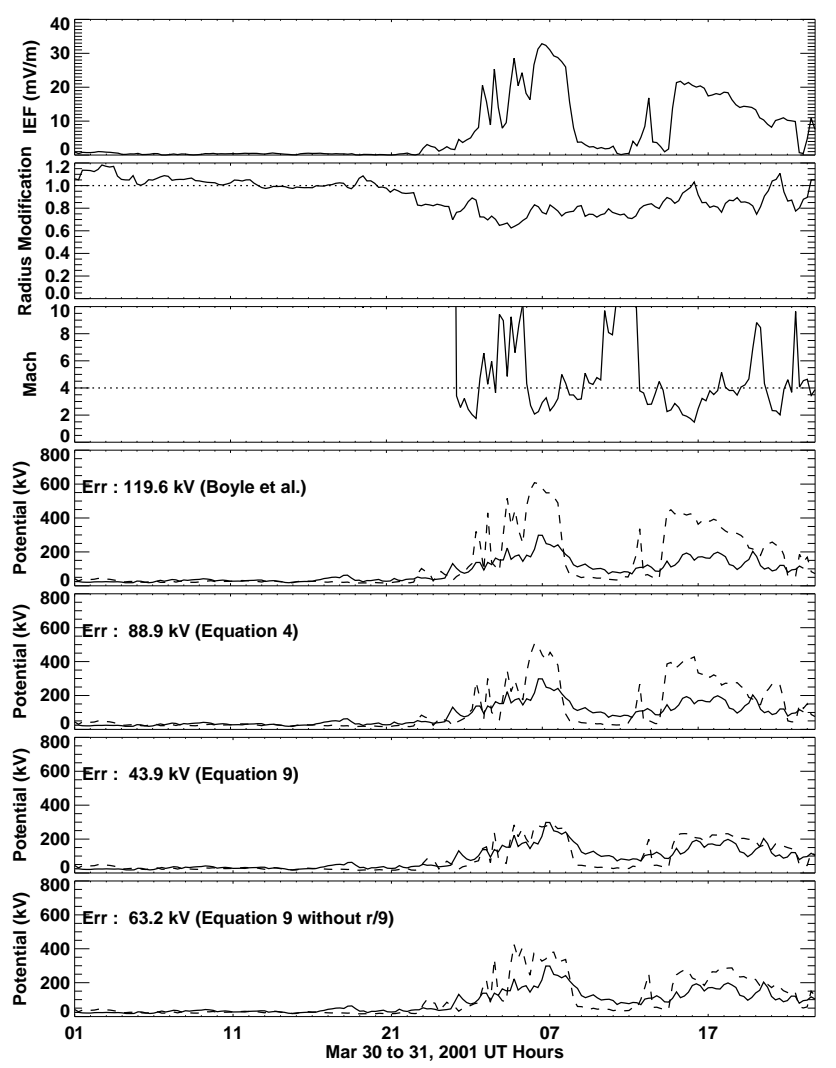

Fig. 9. From top to bottom: the reconnection electric field, using Eq. (2); the magnetospheric radius divided by 9; the solar wind Alfvén Mach number; the AMIE cross polar cap potential (CPCP, solid) and the estimated CPCP using Eq. (4); and the AMIE CPCP (solid) and the estimated CPCP using Eq. (9). The time period is 30-31 March 2001.

field. Reiff et al. (1981) show that they can increase the correlation between the IMF and the cross polar cap potential by "shocking" the IMF up to a certain level and having values above that be constant.

Recently, Lopez et al. (2004) discuss the role of the solar wind number density in controlling the strength of the cross polar cap potential and ionospheric Joule heating. They show that the solar wind density and the magnetic field strength control the compression ratio of the bow shock. During nominal solar wind and IMF conditions, the magnetic field is always increased by a constant factor (independent of the solar wind density) as it goes through the shock. As the magnetic field increases, the solar wind density gains more control over the shock compression.

This can be quantified if one only considers magnetic fields that are tangential to the bow shock (i.e., only $B_{y}$ and $B_{z}$ components of the IMF). The following set of equations can be used to determine the increase in field strength and density across the bow shock (Roberge and Draine, 1993):

$$
\begin{aligned}
p_{u} & =n_{u} k T_{u} \\
C_{s u} & =\sqrt{\frac{\gamma p_{u}}{\rho_{u}}}
\end{aligned}
$$
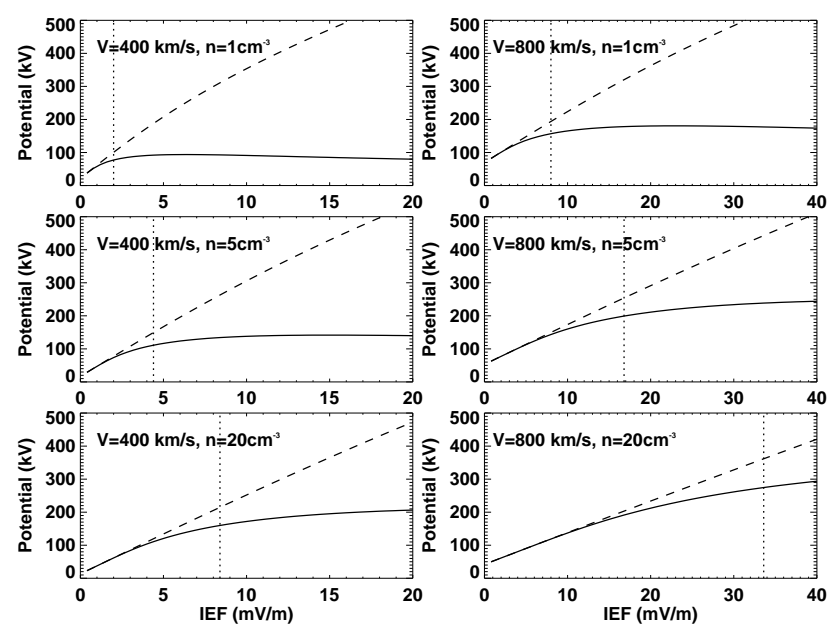

Fig. 10. Examples of CPCP curves as a function of REF using Eq. (9) (solid) and Eq. (4) (dashed). The left curves use an input solar wind velocity of $400 \mathrm{~km} / \mathrm{s}$, while the right curves use $800 \mathrm{~km} / \mathrm{s}$. From top to bottom, the input solar wind density is changed from $1 \mathrm{~cm}^{-3}$ to $5 \mathrm{~cm}^{-3}$ to $20 \mathrm{~cm}^{-3}$. The vertical line indicates when the solar wind Mach number is four. Points to the right are less than four. It should be noted that the vertical scales are the same on all plots, but the left plots stop at $\mathrm{REF}=20 \mathrm{mV} / \mathrm{m}$, while the right plots stop at $\mathrm{REF}=40 \mathrm{mV} / \mathrm{m}$. The REF is altered by changing the magnitude of the IMF $B_{z}$ component - there is no $B_{y}$ component.

$$
\begin{aligned}
C_{A u} & =\frac{B_{u}}{\sqrt{\mu_{0} \rho_{u}}} \\
M_{s u} & =\frac{V_{u}}{C_{s u}} \\
M_{A u} & =\frac{V_{u}}{C_{A u}} \\
C & =\gamma-1+2 M_{s u}^{-2}+\gamma M_{A u}^{-2} \\
\frac{\rho_{d}}{\rho_{u}} & =\frac{B_{d}}{B_{u}}=\frac{2(\gamma+1)}{C+\sqrt{C^{2}+4(\gamma+1)(2-\gamma) M_{A u}^{-2}}},
\end{aligned}
$$

where symbols with a subscript " $u$ " are upstream of the bow shock, and values with a subscript " $d$ " are downstream of the bow shock. $T$ is the temperature of the solar wind, $k$ is Boltzmann's constant, $\gamma$ is $5 / 3, n_{u}$ is the solar wind number density, $\rho_{u}$ is the solar wind mass density, $p_{u}$ is the kinetic pressure of the solar wind, $C_{s u}$ is the solar wind sound speed, $C_{A u}$ is the solar wind Alfvén speed, $V_{u}$ is the solar wind speed, and $M_{A u}$ and $M_{s u}$ are the Alfvén and sonic Mach numbers, respectively.

An important consideration in this formulation is that it is for a shock in which $\boldsymbol{B}$ is perpendicular to the shock normal. This is only true (at the subsolar point) when $B_{x}=0$. When $B_{x}$ is non-zero, this formulation will be incorrect for the subsolar point. The relative strength of $B_{x}$ to $\sqrt{B_{y}^{2}+B_{z}^{2}}$ will determine how relevant it is. During large events in which the main components of the IMF are in $B_{z}$ and $B_{y}$, this formulation holds true. 


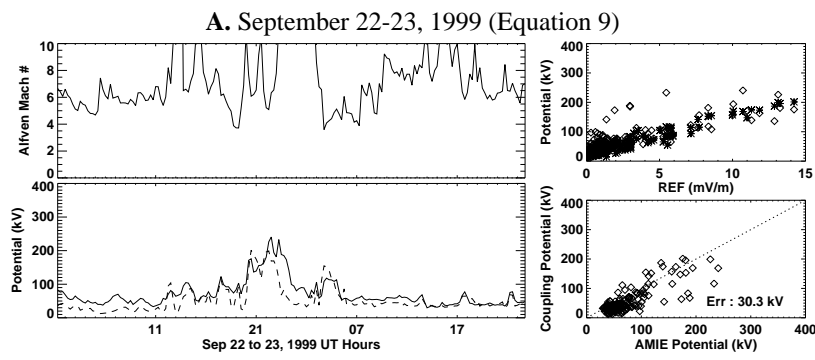

B. October 21-22, 1999 (Equation 9)

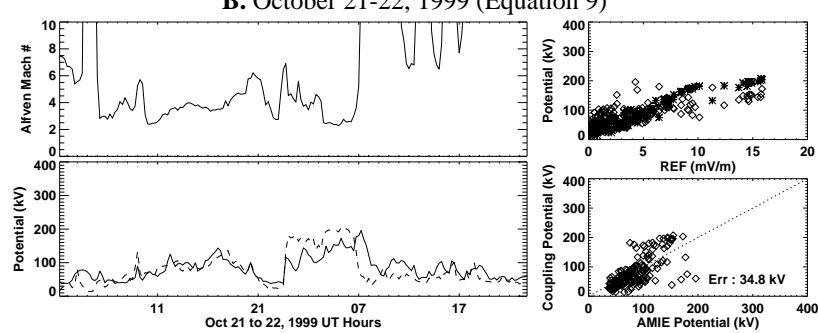

C. April 4-5, 2000 (Equation 9)

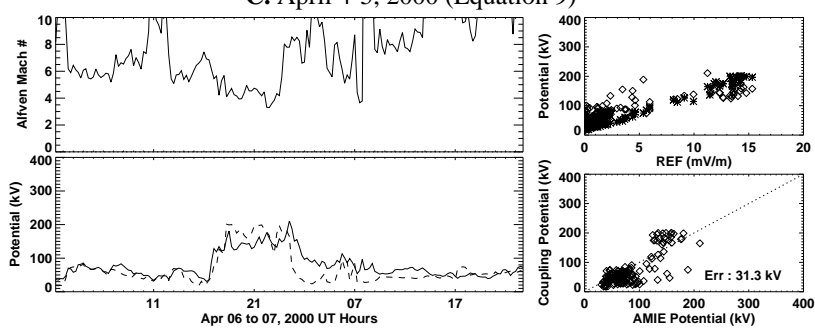

Fig. 11. The same three events in Fig. 1, plotted in the same way, except Eq. (9) was used rather than estimating the CPCP with Boyle et al. (1997). The top plot is the Alfvén Mach number.

Typically, the sound speed in the solar wind is on the order of $50 \mathrm{~km} / \mathrm{s}$, depending on the solar wind temperature. This means that the sound Mach number is on the order of 7 to 20 (given solar wind speeds of $350 \mathrm{~km} / \mathrm{s}-1000 \mathrm{~km} / \mathrm{s}$ ). The Alfvén Mach number is typically in the range of eight for nominal solar wind and IMF conditions. These large values of $M_{A u}$ and $M_{s u}$ imply that:

$B_{d} \simeq \frac{\gamma+1}{\gamma-1} B_{u}=\frac{5 / 3+1}{5 / 3-1} B_{u}=4 B_{u}$.

The magnetosheath magnetic field is approximately four times the IMF for tangential fields and nominal solar wind conditions.

Figure 15 shows the relationship between the IMF and the solar wind Alfvén Mach number for a number of different solar wind number densities. The grey shaded region is considered nominal values (i.e., $2.5 \mathrm{~cm}^{-3}<n_{u}<10 \mathrm{~cm}^{-3}$ and $1 \mathrm{nT}<B_{u}<10 \mathrm{nT}$ ). In this regime, the Alfvén Mach number is always above three. It is clear that, as the number density of the solar wind decreases, the Mach number also decreases, meaning that the solar wind can become subAlfvénic at lower magnetic field values. For example, with a number density of $1 \mathrm{~cm}^{-3}$, an $18 \mathrm{nT}$ magnetic field means a sub-Alfvénic solar wind (if the solar wind speed is $400 \mathrm{~km} / \mathrm{s}$ ).

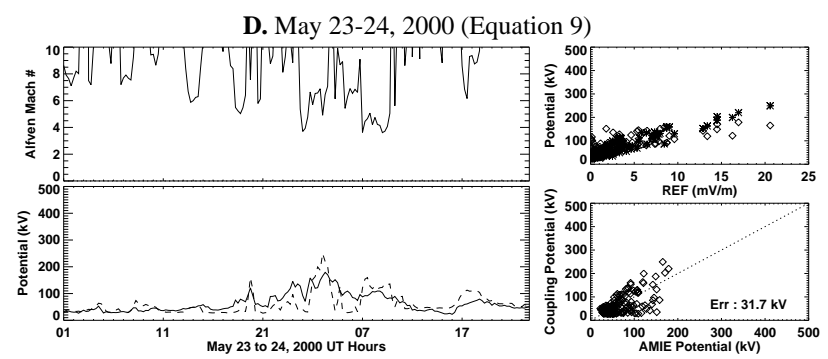

E. July 15-16, 2000 (Equation 9)

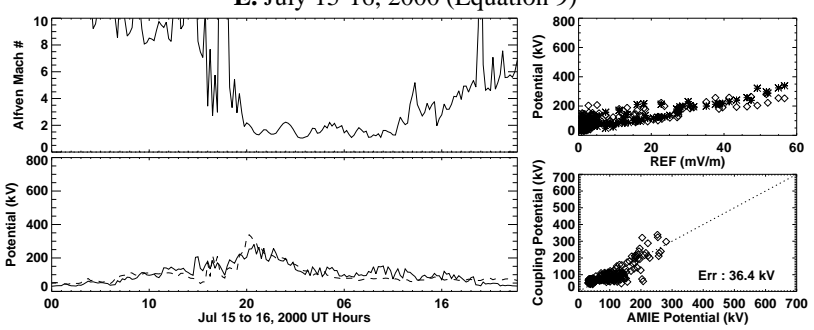

F. August 11-12, 2000 (Equation 9)

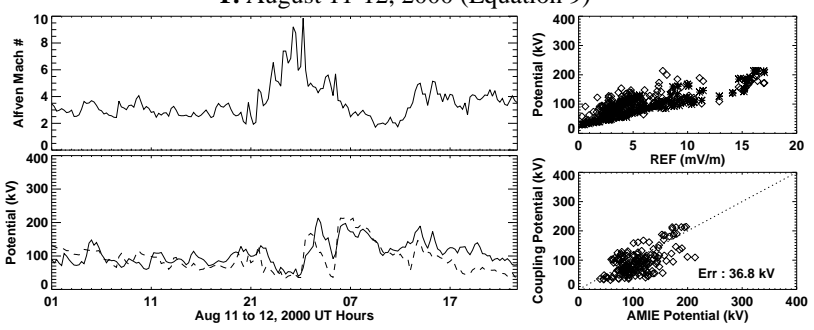

Fig. 12. The same three events in Fig. 2, plotted in the same way as Fig. 11.

When the solar wind number density is $25 \mathrm{~cm}^{-3}$, the solar wind becomes sub-Alfvénic only when the IMF is larger than $90 \mathrm{nT}$, which is a very rare occurrence. When one considers that the cores of magnetic clouds are regions of high magnetic field strength, low temperature, and low density, they are in the exact region that can easily become sub-Alfvénic. These are also the times in which saturation of the CPCP occurs.

Figure 16 offers a possible explanation for the saturation of the cross polar cap potential. This plot shows the shocked (i.e., magnetosheath) magnetic field strength as a function of the upstream magnetic field strength for a number of different solar wind number densities. If one of the lines is followed, there is a sharp, linear rise of the magnetic field when the Alfvén Mach number is very large (i.e., $B_{u}$ is small). This line is simply $B_{d}=4 B_{u}$. As the Mach number decreases below three, the sheath field saturates at around $B_{d}=2 B_{u}$, and actually starts to decrease. When the Mach number passes below one, there is no longer a shock, so Eq. (16) is no longer valid.

Equation (9) multiplies the magnetic field of the Boyle et al. (1997) formulation by a factor of $\left(1-e^{-M_{a} / 3}\right)$, which has a very similar dependence on the Alfvén Mach number as Eq. (16). Figure 17 shows the ratio of the downstream and upstream magnetic fields Eq. (16) as a function of upstream 

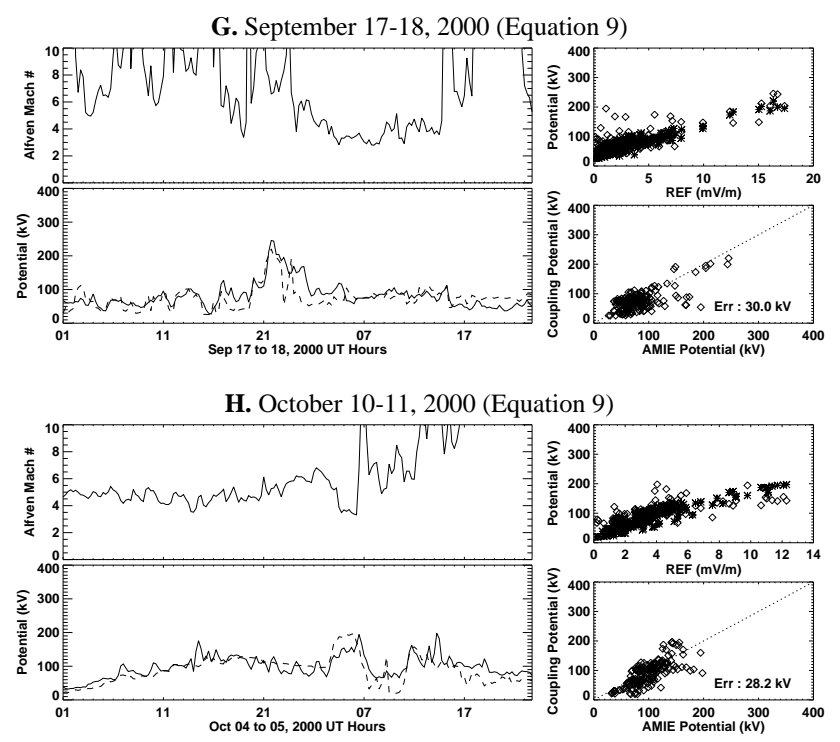

I. November 6-7, 2000 (Equation 9)

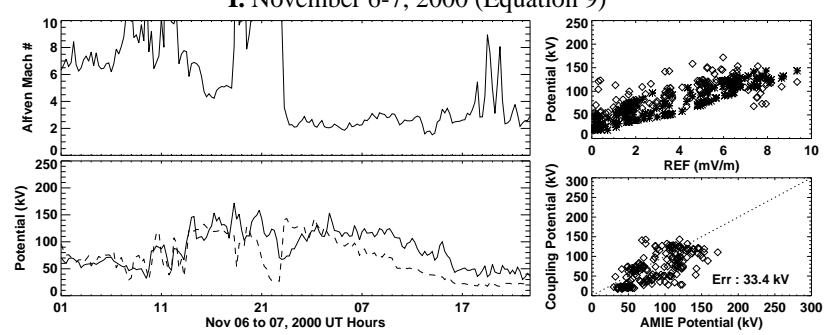

Fig. 13. The same three events in Fig. 3, plotted in the same way as Fig. 11.

magnetic field. In addition, $4\left(1-e^{-M_{a} / 3}\right)$ is over-plotted to show that the lines almost overlay each other. This means that by taking into account the shocking of the solar wind, either with Eq. (16) (divided by four), or with a simple exponential dependence, the saturation of the cross polar cap potential can be accurately modeled.

It should be noted that the solar wind velocity decreases in speed by the same ratio as the magnetic field through the shock, meaning that the electric field remains the same through the shock. At the subsolar point though, the velocity decreases to zero as it approaches the magnetopause (independent of the shock strength), while the magnetic field increases to some value that is most likely controlled by the shocked magnetic field strength. The original Boyle et al. (1997) formulation does not contain the velocity in the primary coupling term, so the decrease in the velocity through the shock need not be compensated for in this term. The viscous interaction term, on the other hand, does have a $v^{2}$ term. It is not reduced in the formulation above because the viscous interaction takes place on the sides of the magnetosphere, after the solar wind has accelerated back up to some significant fraction of the original velocity.

Recently it has been shown that during time periods of low Alfven Mach numbers, the magnetosphere can exhibit global sawtooth oscillations (J. Borovsky, personal commu-
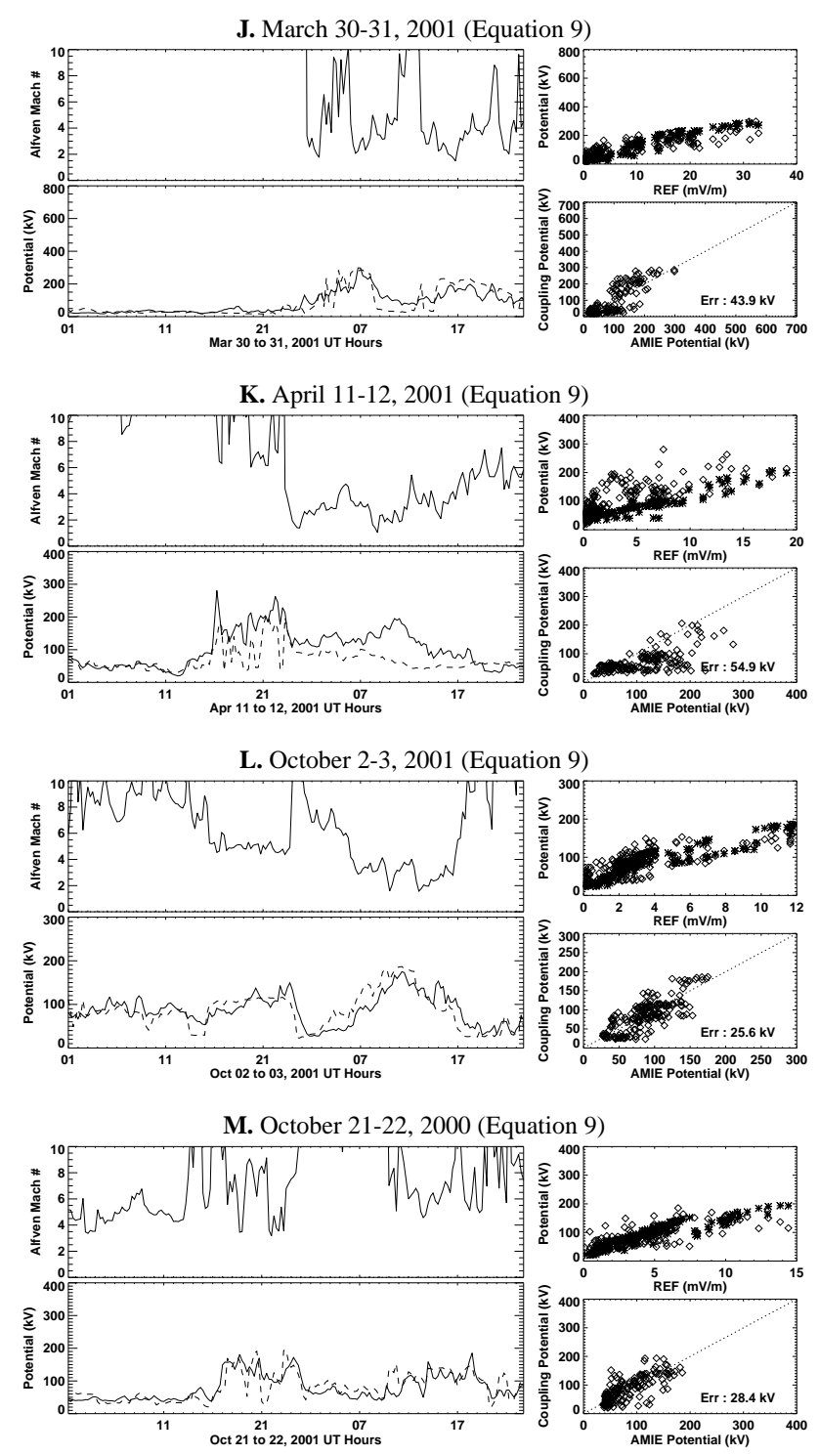

Fig. 14. The same four events in Fig. 4, plotted in the same way as Fig. 11.

nication). It could be possible that these two phenomena both occur during similar driving conditions, and may both be ramifications of the different coupling that may occur between the IMF and the magnetosphere during low Alfvén Mach number conditions.

Obviously, this idea fits quite well with the idea proposed by Reiff et al. (1981), but it is different than other ideas of what causes the saturation of the ionospheric CPCP. One of the most popular ideas on why the CPCP saturates was put forth by Siscoe et al. (2002).

\subsection{Siscoe-Hill Formulation}

In a study conducted by Hill et al. (1976), it was theorized that the observation of high energy particles in the magnetosphere of Mercury, and the lack of high energy particles in 


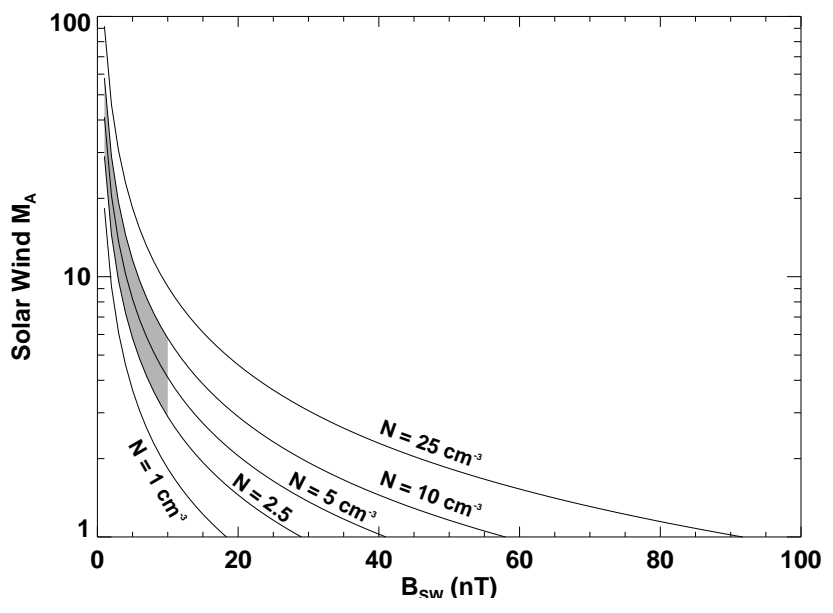

Fig. 15. The solar wind Alfvén Mach number as a function of the IMF strength for a number of solar wind number densities. The solar wind speed is $400 \mathrm{~km} / \mathrm{s}$ in this plot. The shaded region represents typical values of the solar wind number density and IMF strength.

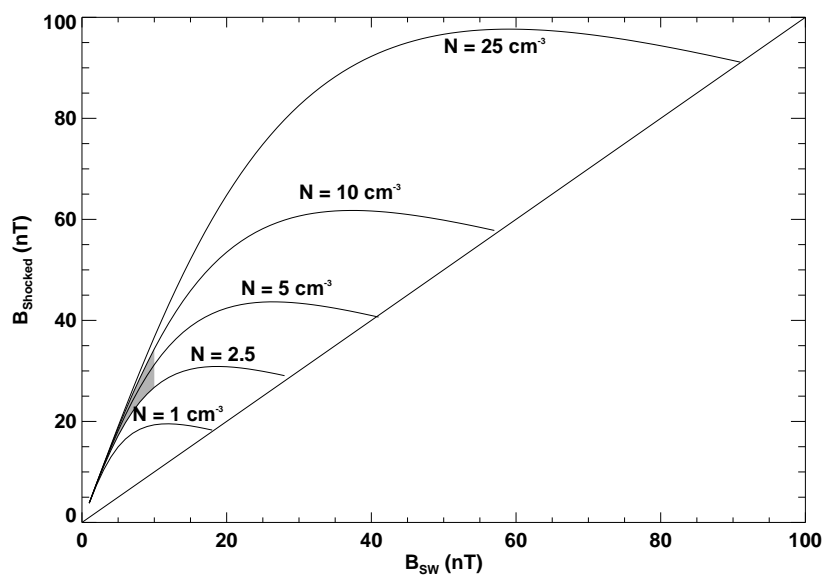

Fig. 16. The magnetosheath magnetic field strength as a function of the upstream IMF strength for a number of solar wind number densities. The bottom right area represents a region in which the Alfvén Mach number is less than one, so it is not considered. The shaded region represents typical values of the solar wind number density and IMF strength. The solar wind speed is $400 \mathrm{~km} / \mathrm{s}$ in this plot.

the magnetosphere of Mars could be explained by considering the ionospheric conductance. They analytically showed that if the conductance is large (as in the Martian system), the high latitude cross polar cap potential can be severely limited. If the conductance is negligible, the potential may be unbounded. Hill et al. (1976) described the polar cap potential:

$\Phi=\frac{\Phi_{m s} \Phi_{\text {iono }}}{\Phi_{m s}+\Phi_{\text {iono }}}$

where $\Phi_{\text {iono }}$ represents a maximum potential that the ionosphere can sustain, $\Phi_{m s}$ is the magnetospheric merging potential similar to that described above, and $\Phi$ is

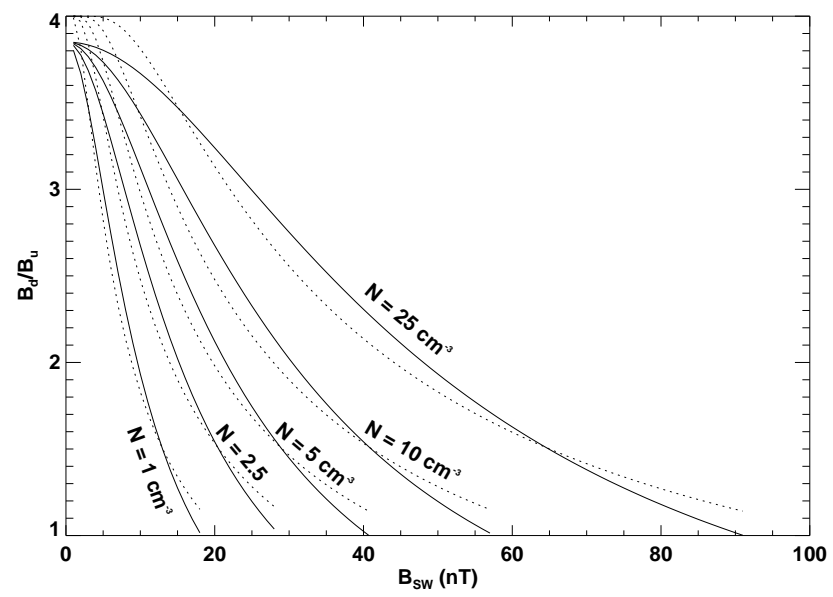

Fig. 17. The ratio of the downstream and upstream magnetic fields as a function of upstream magnetic field strength (i.e., Eq. (16)) are plotted as solid lines for five densities. The formula $4\left(1-e^{-M_{a} / 3}\right)$ is over-plotted as dotted lines for the five different densities.

the ionospheric cross polar cap potential. The Hill et al. (1976) formulation shows that the true ionospheric potential is a combination of the magnetospheric merging potential and the amount of potential that the ionosphere can sustain. Hill et al. (1976) show that for Mercury, which has no ionosphere, $\Phi_{\text {iono }}$ is very large, but $\Phi_{m s}$ is small, so $\Phi=\Phi_{m s}$. On Mars, the situation is reversed - the ionospheric potential is small, while the magnetospheric merging potential is (relatively) large, so $\Phi=\Phi_{\text {iono. }}$. On Earth, it is argued, these potentials are similar to each other during nominal conditions.

Reiff et al. (1981) showed that the cross polar cap potential of Earth could be predicted quite accurately using a modification of the Hill et al. (1976) formulation. Siscoe et al. (2002) also modified this formulation and showed that it could be used to determine how the ionospheric cross polar cap potential will saturate for strong interplanetary electric fields (i.e. $\Phi_{m s}$ becoming much larger than $\Phi_{\text {iono }}$, so $\Phi$ pushes towards $\Phi_{\text {iono }}$ ). They explain that the saturation occurs when the region 1 current system causes a significant perturbation (i.e. $50 \%$ of the dipole field) at the subsolar magnetopause.

Equation (13) of the Siscoe et al. (2002) study (Eq. (1), above) relates the ionospheric cross polar cap potential to the electric field and pressure in the solar wind, the IMF clock angle, the dipole strength, and the ionospheric conductance. There are aspects of the Siscoe et al. (2002) formulation that are similar to ideas put forth here. Namely that the CPCP is an integral of the electric field over some length that is determined by the pressure in the solar wind. In addition, there is a geometrical term that describes the efficiency of the reconnection $F(\theta)$ that is similar to one used in the Boyle et al. (1997) formulation.

This study argues that the saturation of the cross polar cap potential can be explained by phenomena external to the magnetosphere. It is argued that the internal properties of the solar wind and its interaction with a magnetized (or 
conducting) body determine the point at which saturation occurs. This is fundamentally different than the Siscoe et al. (2002) study, in which the dipole strength and ionospheric conductance plays a significant role, since these are the internal quantities that help to determine how much current can flow into the ionosphere.

\subsection{The ionospheric conductance}

The ionospheric conductance plays a large role in determining the strength of the ionospheric electric field, and hence the strength of the ionospheric CPCP (Ridley et al., 2004). There is absolutely no question of whether this is true or not. The outstanding question is whether the ionospheric conductance plays a role in determining when the ionospheric potential starts to saturate.

The Boyle et al. (1997) formulation has no conductance term in it. This is somewhat surprising, since one would expect that if the conductance changes by an order of magnitude (as can happen from strong winter conditions to strong summer conditions), the potential would change by an order of magnitude also. This doesn't happen. The magnetospheric currents end up closing mostly through the conducting ionosphere, and the winter ionosphere only receives enough current to somewhat equalize the potentials. Beaujardiere et al. (1991) show that the ionospheric potential changes by approximately $20 \%$ between summer and winter, while the conductance can change by a factor of 20 (Ridley et al., 2004). The relationship between the ionospheric CPCP and the conductance has not been completely quantified.

Ober et al. (2003) show that the conductance plays a role in determining the potential during strong driving conditions (as it does during nominal conditions), but it is unclear if it plays a role in determining when the saturation begins. Figure 4 of Nagatsuma (2004) shows plots of the CPCP estimated from the PCI index versus the merging electric field (MEF) for different conductance levels. Interestingly, all of the PCI estimated CPCP versus MEF curves are linear up to the highest MEF on the plot, while the Siscoe et al. (2002) lines show clear saturation effects.

\subsection{Other causes of saturation}

Siscoe et al. (2004) discuss a number of different mechanisms that may be responsible for the saturation of the CPCP, two of which could be considered external mechanisms, but were couched in terms of internal mechanisms. For example, modeling has shown that when the solar wind electric field reaches a large value, the nose of the magnetosphere forms a dimple. This dimple formation may reduce the effective reconnection, because the magnetic field has to bend inwards towards the reconnection site. This curvature of the field is then in the opposite direction of the flow, so it acts to slow the flow down. Siscoe et al. (2004) argue that this may be caused by the region 1 currents reducing the magnetic field at the nose of the magnetosphere or that reconnection could eat away the subsolar magnetosphere, causing an indenta- tion. In other words, the large reconnection rate could cause an indentation in the magnetopause, inhibiting reconnection, thereby limiting the total amount of reconnection that can take place.

The second external mechanism that is suggested by Siscoe et al. (2004) is quiet similar to the mechanism suggested here. They point out that the Alfvén Mach number decreases as the CPCP saturates, and that the magnetopause becomes more blunt as the Mach number decreases. The bluntness of the magnetopause could either inhibit reconnection or it could allow more of the magnetic field to flow around the magnetosphere without the reconnection process. The question that remains with this mechanism is what actually causes the saturation as the Mach number decreases, which is what is described above.

\subsection{Pressure considerations}

The exact role of the solar wind pressure in determining the ionospheric CPCP is still unknown. In the Siscoe et al. (2002) formulation, the CPCP has different functions of the pressure depending on whether the potential is saturated or not. In the linear regime, the potential is dependent upon $p^{-1 / 6}$ (i.e. $p^{1 / 3} / p^{1 / 2}$ ), similar to what is described above in Eqs. (4) and (5). This implies that as the solar wind pressure increases, the CPCP will decrease, as in the formulation described above. In the saturated regime, the relationship is $p^{1 / 3}$. This means that if the solar wind pressure increases, the CPCP will increase also.

It should be noted that when discussing increases or decreases in the solar wind ram pressure $\left(\rho V^{2}\right)$, the term that is actually changing needs to be described. If the velocity changes, both the pressure and the electric field change, making the relationship with the CPCP more complex. If only the density changes, the electric field stays constant.

Recently, Boudouris et al. (2004) has shown that (for at least one event), the solar wind pressure caused the CPCP to increase dramatically. The Siscoe et al. (2002) formulation did not match the large increase. Obviously, the formulation described here would not match either, since the CPCP would decrease with increasing density. Shepherd et al. (2003) showed that the SuperDARN measured CPCP was roughly independent of the solar wind pressure at all levels of solar wind electric field.

The question then remains as to the role of the solar wind density (or pressure) in determining the strength of the ionospheric CPCP. If we ignore the fact that the magnetopause shrinks with increasing pressure (i.e. take the $r_{m s} / 9$ out of Eq. (9)), some of the events reproduces the AMIE derived potential much better. This is shown Table 1. Comparing columns "Eq. (9)" and "Eq. (9- $\left.R_{m s} / 9\right)$ ", some of the events have a lower error if the magnetospheric size is not included (11 August 2000; 17 September 2000; 6 November 2000; and 11 April 2001). Conversely, a few of the event are reproduced significantly better with the size correction. For example, 30-31 March 2001 is reproduced 16\% better with the size correction. This can also be seen in comparing the 
second to last and the bottom plots of Fig. 9. The CPCP is better modeled using the magnetosphere size correction factor.

It is therefore left up to the reader whether to include the magnetospheric size correction factor $\left(r_{m s} / 9\right)$ in Eq. (9).

\section{Internal vs. external}

There are no trivial ways of statistically determining whether the saturation of the CPCP is caused by an internal or external mechanism. This is because the system very rarely allows for systematic testing: the solar wind velocity, density, and IMF are all related to each other, such that when one changes, the others tend to change. The time periods of saturation tend to not occur during solar minimum, so testing against conductance variations are difficult. So a statistical study would be difficult to conduct.

Besides conducting a statistical study, the most obvious way to test this idea (using data) would be to search for time periods in which the solar wind Mach number is small, but the IMF is also quite low. For example, on 11 May 1999, the Alfvén Mach number decreased to 0.78 , while $B_{z}$ only decreased to $-7 \mathrm{nT}$. This is because the density decreased to extremely low values. We present this event in Fig. 18. This is in the same format as Fig. 9, but showing the 1112 May 1999 event. The REF never exceeds $4 \mathrm{mV} / \mathrm{m}$, and only exceeds $3 \mathrm{mV} / \mathrm{m}$ for a few minutes. Because the solar wind pressure is so low, the radius modification factor is very high, actually elevating the potential throughout most of the interval, instead of decreasing it. When comparing the Boyle et al. (1997) formulation to Eq. (4), the potential is better modeled, because the potential is increased up to the AMIE values. When Eq. (9) is used without the radius modification factor (i.e. the bottom plot), the time between 04:00-10:00 UT on 12 May is much better modeled, but the modeled potential before this time is too low. Using Eq. (9), the potential is modeled pretty well everywhere, except there is an overestimate between 04:00-10:00 UT.

Does this prove that the external mechanism more accurately describes the system than an internal mechanism? If one considers that one the main ideas of the internal mechanism is that the region 1 currents deforms the nose of the magnetosphere, it is hard to believe that this can occur when the cross polar cap potential never goes above $80 \mathrm{kV}$, and the magnetopause stand-off distance is between 13 and $18 R_{E}$. So, the very fact that the drivers are so small leads one to believe that no internal magnetospheric process should be saturated, yet it shows signs of saturation (between 04:0010:00 UT on 12 May). The Mach number is significantly reduced during that time period, though, pointing to the external mechanism. Interestingly, with similar REF drivers towards the end of the 12th, there are no signs of saturation. During this time, the Mach number climbed back to nominal solar wind values.

Besides examining data that could be interpreted many different ways, modeling offers a good way to systematically test the solar wind - magnetosphere coupling. Here are some possible tests that can be done:

1. Conduct many simulations varying the IMF $B_{z}$ component between 0 and $-100 \mathrm{nT}$. Change the ionospheric conductance and repeat the experiments. How does varying the ionospheric conductance change the point at which the CPCP starts to become nonlinearly related to the interplanetary electric field?

2. Conduct many simulations varying the IMF $B_{z}$ component between 0 and $-100 \mathrm{nT}$. Change the solar wind density and repeat. How does this change both the level of the potential and the point at which it starts to saturate?

3. Conduct many simulations varying the IMF $B_{z}$ component between 0 and $-100 \mathrm{nT}$. Change the dipole strength and repeat. How does this change both the level of the potential and the point at which it starts to saturate?

4. Attempt to change the reconnection rate at the dayside magnetopause numerically while leaving all other quantities exactly the same. This could be done by adding resistivity to the reconnection site. One could then attempt to determine whether there is a dimple formation, and if so, is there a maximum reconnection rate that can be achieved. Then the question of how the CPCP changes as a function of the reconnection rate can be addressed also.

It is our speculation that the CPCP will always start to saturate at the point in which the Alfvén Mach number decreases below 3-4 independent of internal quantities. Saturation should be reached by the time the Mach number is close to 2 . It is not suggested that the potential will be unchanged, but that the point at which the potential starts to saturate should not be dependent upon internal quantities.

\section{Conclusions}

In this study we present 13 events in which the reconnection electric field becomes larger than $12 \mathrm{mV} / \mathrm{m}$ for some time period. At some point during all of these 13 events, the ionospheric cross polar cap potential calculated from the Boyle et al. (1997) formulation over-predicts the AMIE CPCP. We show that when the size of the magnetosphere is considered, the modified Boyle et al. (1997) formulation better matches the majority of the events (by 10\%), but in other studies it has been shown that the CPCP should increase or stay relatively constant as the pressure increases (Shepherd et al., 2003; Boudouris et al., 2004).

We further show that during all of the time periods in which the over-prediction of the CPCP occurs, the solar wind Alfvén Mach number is reduced beneath its nominal value. When we take this into account, almost all of the overprediction of the CPCP is accounted for $(28.2 \%$ decrease in 


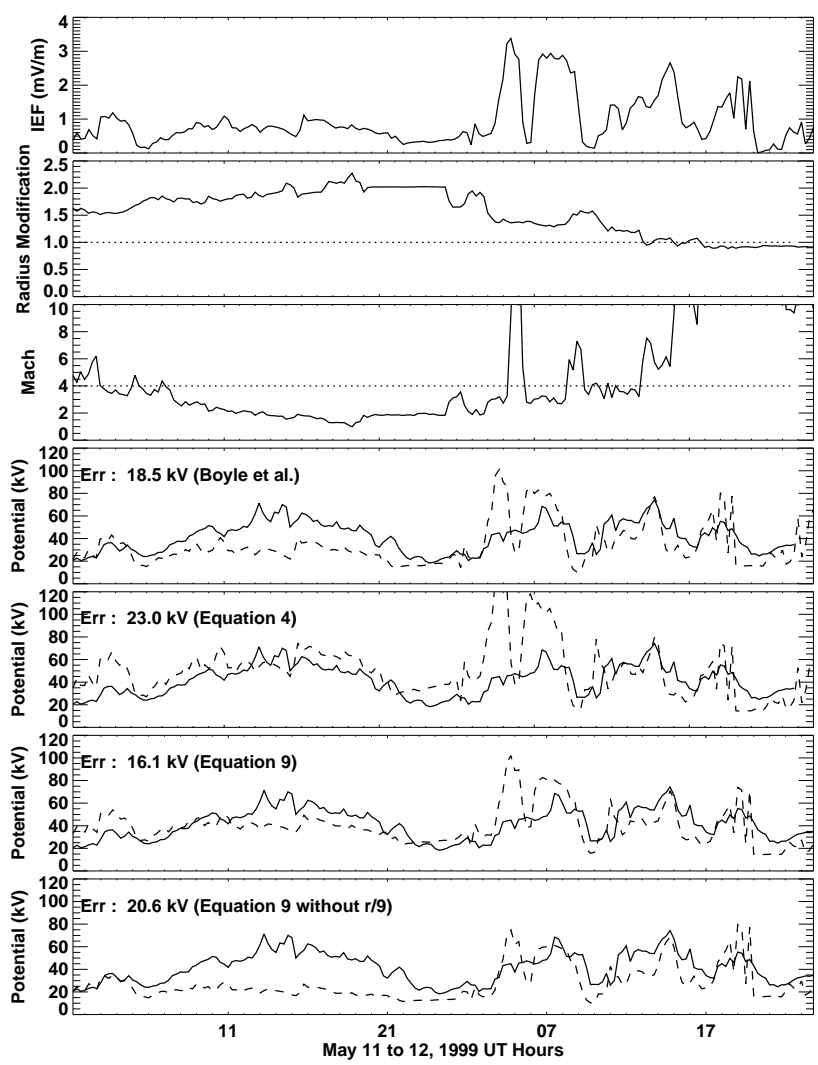

Fig. 18. May 11-12, 1999, in the same format as Fig. 9.

the error when only the Mach number is considered, 33.4\% decrease in the error when both the Mach number and magnetospheric size are considered). We therefore conclude that the solar wind Alfvén Mach number may play a significant role in the magnetosphere solar wind coupling.

If the magnetosheath magnetic field is considered to be more important than the actual solar wind magnetic field in the coupling between the solar wind and the magnetosphere, then the compression of the magnetic field across the bow shock must be considered. We show that for nominal conditions, the magnetic field is increased by almost a factor of four across the bow shock. Under stronger magnetic field conditions (i.e., smaller Alfvén Mach numbers), the compression is reduced below the nominal increase of four, reaching an increase of two when the Mach number goes below three. When this is taken into account, the CPCP can be expressed as $\Phi=\left(10^{-4} v^{2}+11.7 B\left(1-e^{-M_{a} / 3}\right) \sin ^{3}(\theta / 2)\right) \frac{r_{m s}}{9}$. This is a simple modification of the original Boyle et al. (1997) formulation, and explains the saturation of the cross polar cap potential during these intervals. Because the role of the solar wind density in determining the strength of the CPCP is still questionable, we leave it up to the reader whether to include the $\frac{r_{m s}}{9}$ magnetospheric size correction factor.

Arguing that the solar wind Alfvén Mach number can control the saturation of the ionospheric cross polar cap potential suggests that the saturation is caused by a process external to the magnetosphere, while Siscoe et al. (2002) argue that the saturation is caused by processes internal to the magnetosphere. Further studies are needed to determine whether the saturation is caused by an internal or external mechanism.

Acknowledgements. I wish to thank G. Tòth and K. C. Hansen in their helpful discussions on shock compressions. The ACE solar wind and IMF data was downloaded from the NASA CDAWEB FTP site. This research was supposed by NSF through grants ATM0077555 and ATM-0417839 and the DoD MURI program grant F4960-01-1-0359.

Topical Editor T. Pulkkinen thanks V. Vasyliunas and another referee for their help in evaluating this paper.

\section{References}

Ahn, B.-H., Akasofu, S.-I., Kamide, Y., and King, J.: Cross-polar cap potential drop and the energy coupling function, J. Geophys. Res., 89, 11, 028-11 032, 1984.

Beaujardiere, O. D. L., Alcayde, D., Fontanari, J., and Leger, C.: Seasonal Dependence of High-Latitude Electric Fields, J. Geophys. Res., 96, 5723-5735, 1991.

Bekerat, H., Schunk, R., Scheirles, L., and Ridley, A.: DMSP Comparisons to AMIE, J. Atmos. Sol-Terr. Phys., in press, 2005.

Boudouris, A., Zesta, E., Lyons, L., and Anderson, P.: Evaluation of the Hill-Siscoe transpolar potential saturation model during a solar wind dynamic pressure pulse, Geophys. Res. Lett., 31, L23 802, doi:10.1029/2004GL021252, 2004.

Boyle, C., Reiff, P., and Hairston, M.: Empirical polar cap potentials, J. Geophys. Res., 102, 111-126, 1997.

Friis-Christensen, E., Kamide, Y., Richmond, A., and Matsushita, S.: Interplanetary magnetic field control of high-latitude electric fields and currents determined from Greenland magnetometer chain, J. Geophys. Res., 90, 1325-1338, 1985.

Hairston, M., Hill, T., and Heelis, R.: Observed saturation of the ionospheric polar cap potential during the 31 March 2001 storm, Geophys. Res. Lett., 30, 1325, doi:10.1029/2002GL015894, 2003.

Hill, T., Dessler, A., and Wolf, R.: Mercury and Mars: The role of ionospheric conductivity in the acceleration of magnetospheric particles, Geophys. Res. Lett., 3, 429-432, 1976.

Kan, J. and Lee, L.: Energy coupling function and solar wind magnetosphere dynamo, Geophys. Res. Lett., 6, 577-580, 1979.

ihn, E. A., Redmon, R., and Ridley, A.: A statistical comparison of the AMIE derived and DMSP-SSIES observed high-latitude ionospheric electric field, J. Geophys. Res., in press, 2005.

Liemohn, M. W. and Ridley, A. J.: Comment on "Nonlinear response of the polar ionosphere to large values of the interplanetary electric field" by C.T. Russell et al., J. Geophys. Res., 107, 1460, doi:10.1029/2002JA009440, 2002.

Lopez, R., Wiltberger, M., Hernandez, S., and Lyon, J.: Solar wind density control of energy transfer to the magnetopause, Geophys. Res. Lett., in press, 2004.

Merkine, V., Papadopoulos, K., Milikh, G., Sharma, A., Shao, X., Lyon, J., and Goodrich, C.: Effects of the solar wind electric field and ionospheric conductance on the cross polar cap potential: Results of global MHD modeling, Geophys. Res. Lett., 30, 2180, doi:10.1029/2003GL017903, 2003.

Nagatsuma, T.: Saturation of polar cap potential by intense solar wind electric fields, Geophys. Res. Lett., 29, 1422, 10.1029/2001GL014202, 2002. 
Nagatsuma, T.: Conductivity dependence of cross-polar potential saturation, J. Geophys. Res., 109, A04210, doi:10.1029/2003JA010286, 2004.

Ober, D., Maynard, N., and Burke, W.: Testing the Hill model of transpolar saturation, J. Geophys. Res., 108, 1467, doi:10.1029/2003JA010154, 2003.

Papitashvili, V. and Rich, F.: High-latitude ionospheric convection models derived from Defense Meteorological Satellite Program ion drift observations and parameterized by the interplanetary magnetic field strength and direction, J. Geophys. Res., 107, doi:10.1029/2001JA000264, 2002.

Papitashvili, V., Belov, B., Faermark, D., Feldstein, Y., Golyshev, S., Gromova, L., and Levitin, A.: Electric potential patterns in the northern and southern polar regions parameterized by the interplanetary magnatic field, J. Geophys. Res., 99, 13 251-13 262, 1994.

Raeder, J., McPherron, R., Frank, L., Kokubun, S., Lu, G., Mukai, T., Paterson, W., Sigwarth, J., Singer, H., and Slavin, J.: Global simulation of the Geospace Environment Modeling substorm challenge event, J. Geophys. Res., 106, 381-396, 2001.

Reiff, P., Spiro, R., and Hill, T.: Dependence of polar cap potential on interplanetary parameters, J. Geophys. Res., 86, 7639-7648, 1981.

Richmond, A. and Kamide, Y.: Mapping Electrodynamic features of the high-latitude ionosphere from localized observations: Technique, J. Geophys. Res., 93, 5741, 1988.

Ridley, A.: Estimation of the uncertainty in timing the relationship between magnetospheric and solar wind processes, J. Atmos. Sol-Terr. Phys., 62, 757-771, 2000.

Ridley, A. and Kihn, E.: Polar cap index comparisons with AMIE cross polar cap potential, electric field, and polar cap area, Geophys. Res. Lett., 31, doi:10.1029/2003GL019 113, 2004.

Ridley, A., Clauer, C., Lu, G., and Papitashvili, V.: A statistical study of the ionospheric convection response to changing interplanetary magnetic field conditions using the assimilative mapping of ionospheric electrodynamics technique, J. Geophys. Res., 103, 4023-4040, 1998.

Ridley, A., Crowley, G., and Freitas, C.: A empirical model of the ionospheric electric potential, Geophys. Res. Lett., 27, 36753678, 2000.
Ridley, A., Gombosi, T., and Zeeuw, D. D.: Ionospheric control of the magnetospheric configuration: Conductance, Ann. Geophys., 22, 567, 2004,

SRef-ID: 1432-0576/ag/2004-22-567.

Roberge, W. and Draine, B.: A new class of solutions for intersteller magnetohyrodynamic shock waves, Astrophysical J., 350, 700721, 1990.

Russell, C., Lu, G., and Luhmann, J.: Lessons from the ring current injection during the 24-25 September 1998 storm, Geophys. Res. Lett., 27, 1371-1374, 2000.

Russell, C., Luhmann, J., and Lu, G.: The non-linear response of the polar ionosphere to large values of the interplanetary magnetic field, J. Geophys. Res., 106, 18,495, 2001.

Shepherd, S., Ruohoniemi, J., and Greenwald, R.: Cross polar cap potentials measured with Super Dual Auroral Radar Network during quasi-steady solar wind and interplanetary magnetic field conditions, J. Geophys. Res., 107, doi:10.1029/2001JA000 152, 2002.

Shepherd, S., Ruohoniemi, J., and Greenwald, R.: Testing the Hill model of transpolar potential with Super Dual Auroral Radar Network observations, Geophys. Res. Lett., 30, doi:10.1029/2002GL015 426, 2003.

Siscoe, G., Raeder, J., and Ridley, A. J.: Transpolar Potential Saturation Models Compared, J. Geophys. Res., 109, doi:10.1029/2003JA010318, 2004.

Siscoe, G. L., Erickson, G., Sonnerup, B., Maynard, N., Schoendorf, J., Siebert, K., Weimer, D., White, W., and Wilson, G.: Hill model of transpolar potential saturation: Comparisons with MHD simulations, J. Geophys. Res., 107, 1321, doi:10.1029/2001JA009176, 2002.

Sonnerup, B.: Magnetopause reconnection rate, J. Geophys. Res., 79, 1546-1549, 1974.

Troshichev, O., Hayakawa, H., Matsuoka, A., Mukai, T., and Tsuruda, K.: Cross polar cap diameter and voltage as a function of $P C$ index and interplanetary quantities, J. Geophys. Res., 101, 13,429-13 436, 1996.

Weimer, D., Reinleitner, L., Kan, J., Zhu, L., and Akasofu, S.-I.: Saturation of the auroral electrojet current and the polar cap potential, J. Geophys. Res., 95, 18 891-18 987, 1990. 